\title{
Needle-free injection into skin and soft matter with highly focused microjets
}

\author{
Yoshiyuki Tagawa ${ }^{1, *}$, Nikolai Oudalov ${ }^{1}$, A. El Ghalbzouri ${ }^{2}$, Chao Sun $^{1, *}$, and Detlef Lohse ${ }^{1, *}$ \\ ${ }^{1}$ Physics of Fluids Group, MESA Institute and Faculty of Science and Technology, \\ University of Twente, P.O. Box 217, 7500 AE Enschede, Netherlands. \\ ${ }^{2}$ Department of Dermatology, Leiden University Medical Center, Leiden, The Netherlands \\ *Email addresses for correspondence: y.tagawa@utwente.nl, c.sun@utwente.nl, d.lohse@utwente.nl,
}

\begin{abstract}
The development of needle-free drug injection systems is of great importance to global healthcare. However, in spite of its great potential and research history over many decades, these systems are not commonly used. One of the main problems is that existing methods use diffusive jets, which result in scattered penetration and severe deceleration of the jets, causing frequent pain and insufficient penetration. Another longstanding challenge is the development of accurate small volume injections. In this paper we employ a novel method of needle-free drug injection, using highly-focused high speed microjets, which aims to solve these challenges. We experimentally demonstrate that these unique jets are able to penetrate human skin: the focused nature of these microjets creates an injection spot smaller than a mosquito's proboscis and guarantees a high percentage of the liquid being injected. The liquid substances can be delivered to a much larger depth than conventional methods, and create a well-controlled dispersion pattern. Thanks to the excellent controllability of the microjet, small volume injections become feasible. Furthermore, the penetration dynamics is studied through experiments performed on gelatin mixtures (human soft tissue equivalent) and human skin, agreeing well with a viscous stress model which we develop. This model predicts the depth of the penetration into both human skin and soft tissue. The results presented here take needle-free injections a step closer to widespread use.
\end{abstract}

\section{INTRODUCTION}

The development of needle-free drug injection systems is an essential part of the global fight against the spread of disease [1 3]. Contamination, needle-stick injuries [4], painful injections, and needle phobia [5] are issues related to traditional syringe injections with needles that demand attention. Needle-free injections systems offer the prospect of resolving these problems 6 . Previous studies have explored the possibilities of needle-free injections, but important limitations still need to be addressed [7-12.

The main issue that is limiting applicability is the shape of the jets produced by the current systems. These devices create diffusive jets, leading to a large dispersion pattern and unreliable penetration. This in turn creates problems for patients, in the form of frequent bruising and pain [10]. Another problem of conventional methods is that due to the small size of the nozzle diameter, it can easily get clogged, causing disruptions to controllability.

Very recently, we have managed to generate thin, focused microjets with velocities of up to $850 \mathrm{~m} / \mathrm{s}$ by the rapid vaporization of a small mass of liquid in an open liquid-filled capillary as reported in [13. Our novel method of jet creation addresses the issues mentioned above. Ultra-high velocities (more than $200 \mathrm{~m} / \mathrm{s}$ ) combined with a highlyfocused geometry enable one-shot penetration to the desired area and good controllability. The forces exerted on the liquid that is delivered in this way cause minor damage to the medicine that is contained in it [14]. Due to the fine scale of the jet tip $(30 \mu \mathrm{m})$ combined with the high velocities, we can easily adjust the penetration depth according to the requirements. This makes drug delivery efficient and as painless as possible.

In this article we study the penetration dynamics of these highly focused microjets into gelatin mixtures and artificially grown human skin using high-speed imaging. We investigate the penetration depth as a function of the jet velocity and the capillary tube diameter. The understanding of these dynamics will provide essential insight for the development of needle-free injection devices.

\section{RESULTS AND DISCUSSION}

\section{A. Injection into gelatin}

In order to study the penetration of these microjets, we used gelatin $5 \mathrm{wt} \%$ as a model material. This percentage simulates the properties of soft tissue in the human body [8]. Figure 1 provides the first observation of the temporal evolution of the jet penetration. This visualization is of utmost importance for studying the interaction of the microjet and the human body.

As illustrated in figure 1 the sharp tip of the microjet reaches the material first. The diameter of this tip creates an injection spot $\sim 30 \mu \mathrm{m}$, smaller than a mosquito's proboscis. This thin part of the jet starts digging a hole into the 
material. Thanks to the highly focused geometry, there is no splashing around the penetration spot, which is crucial for medical applications. This is clearly indicated in figure 1 for the snapshots covering $30-100 \mu \mathrm{s}$. The snapshot at $100 \mu \mathrm{s}$ shows a well-controlled dispersion pattern. The width of the hole remains as small as the jet diameter. This is in sharp contrast to the existing methods using diffusive jets, which result in scattered penetration. The low-speed thick part of the jet utilizes the entry point created by the thin jet and is efficiently deposited into the material. The penetration of the tip stops at about $300 \mu$ s while the thick jet part continues to make its way to the deepest part of the hole. At the final snapshot $(1.1 \mathrm{~ms})$ almost all volume of the jet released by the capillary tube is deposited into the soft material. The ultrafast jet tip guarantees a high percentage of the liquid being injected as illustrated in figure 1. The entire process is finished after $1.1 \mathrm{~ms}$.

Figure 3 shows the penetration depth of the microjet generated in a $200 \mu \mathrm{m}$ tube into gelatin $5 \mathrm{wt} \%$ as a function of the jet velocity. This penetration is created by a single shot. The depth linearly increases with the jet velocity, covering depths from several hundred microns at low jet speed to $\sim 1.5$ millimeter when the jet velocity approaches $\sim 250 \mathrm{~m} / \mathrm{s}$. This highlights the versatility of this method, making it adjustable to different skin-properties (e.g. children/adults, different skin types) and to a broad range of medical applications (e.g. insulin injection [15, 16], vaccinations [17 19], or medical tattoos).

To get a quantitative understanding, we compare the present results with various models. A model proposed by Baxter et al. [11] is fitted to this experimental data and presented in the figure. The agreement in the low velocity region is fair. However, this model shows a saturation of the penetration depth for velocities above $200 \mathrm{~m} / \mathrm{s}$. In our experiments we did not observe this trend as can be seen in figure 3 . The difference is likely due to the shape of the jet created using our novel method. The jet shape created by conventional methods using syringe-piston system (e.g. [7, 8, 11]) is diffusive. This shape leads to severe deceleration with jet travel distance (especially for high velocities), which is considered in the model by Baxter et al. [11, resulting in shallow penetration. On the other hand, the highly focused jets in our experiments do not experience this significant deceleration.

To address this discrepancy we consider the relation between initial impact velocity of the jet and the drag force. We observe that the gelatin does not show much deformation and the jet penetrates into gelatin with cylindrical shape (see the snapshots at $100 \mu \mathrm{s}$ in figure 1). We model this phenomena as a cylindrical microjet, normal to the gelatin surface. It creates a cylindrical 'crack' inside the gelatin, which keeps the same circular projection area independent of the depth. Figure 2 shows the schematic sketch of this model. The drag forces on the jet are the viscous shear stress at the jet-gelatin interface and the repulsive force on the area of the cross-section of the jet. We consider two basic force models, representing these two different cases: A viscous stress model and a repulsive force model.

We first introduce the viscous stress model. The viscous shear stress $\tau_{w}$ at the wall is $\mu \partial u / \partial r$, where $\mu$ is the dynamic viscosity of the liquid, $u$ is the liquid velocity component in the direction parallel to the wall, and $r$ is the normal position to the wall. In the present case, the velocity scale and the length scale for $\tau_{w}$ are $v$ and $D$, respectively. In the viscous regime the relationship between the velocity and the drag force per unit mass is:

$$
F_{D}=-c_{v} \cdot v,
$$

where $c_{v}$ is a fitting parameter with the units of inverse time. It is known that gentle deposition (small impact velocity) into the soft matter gives no penetration. The penetration starts when the impact velocity $v_{j e t}$ exceeds a critical velocity $v_{c}$. For $v_{j e t} \geq v_{c}$ the final penetration depth $D_{p}$ is given by:

$$
D_{p}=\frac{1}{c_{v}}\left(v_{j e t}-v_{c}\right) .
$$

The other model considers a repulsive force acting on the jet. The repulsive force is modeled as being proportional to the inertial force of the jet $\left(\sim \rho v^{2}\right)$. In this inertial regime, the relationship between the velocity and the drag force per unit mass is given by:

$$
F_{D}=-c_{i} \cdot v^{2},
$$

where $c_{i}$ is a fitting parameter with dimensions of inverse length. Including the offset due to the critical velocity, we obtain the final penetration depth $D_{p}$ as:

$$
D_{p}=\frac{1}{c_{i}} \ln \left(\frac{v_{j e t}}{v_{c}}-1\right)
$$

Both models are compared with the experimental results in figure 3 . It shows that the viscous stress model gives the best agreement, indicating that the jet likely experiences shear stress in the material. This model gives predictive power to our novel method, enabling us to link the physical parameters of our lab experiments to real world medical applications. 


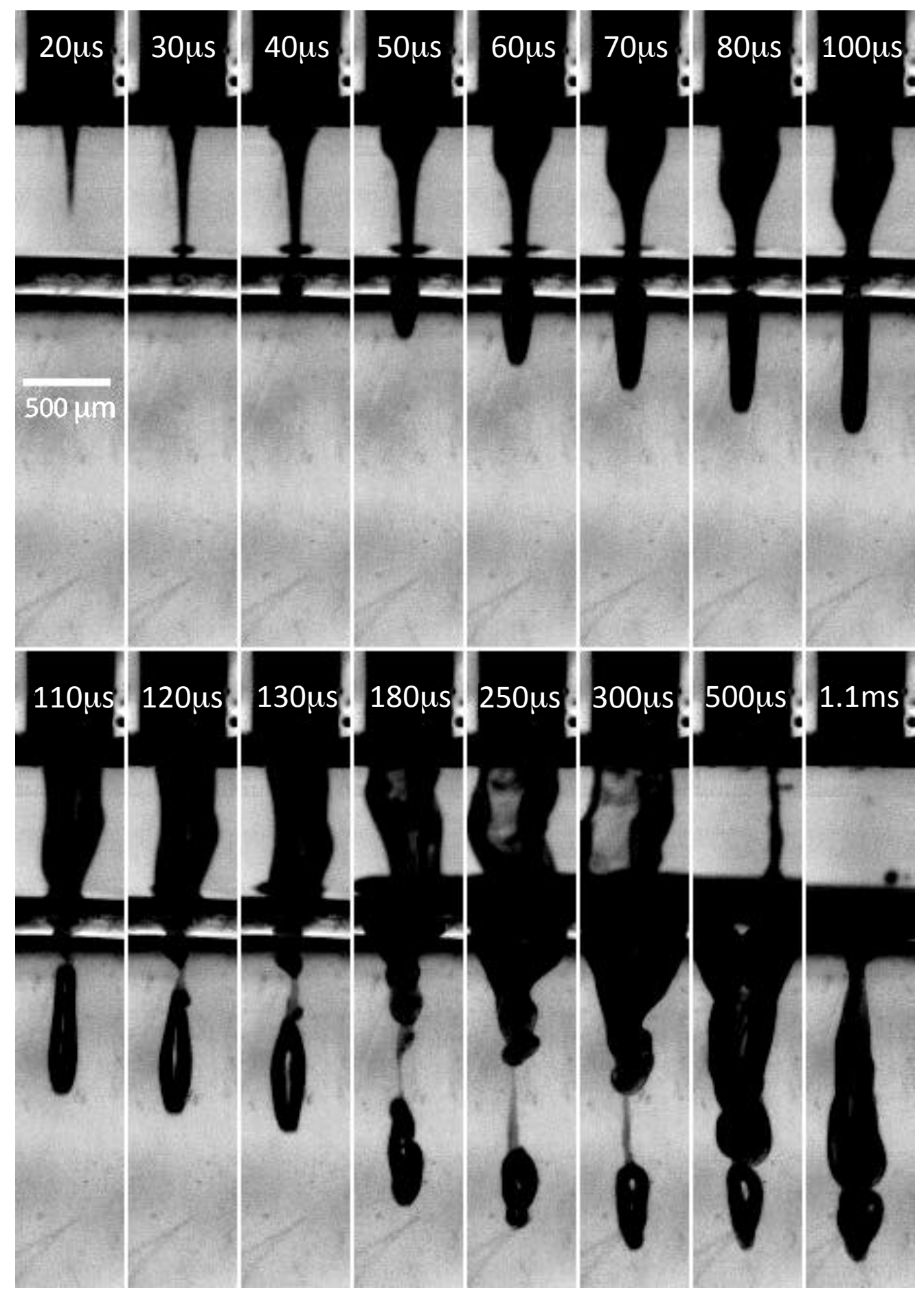

FIG. 1: Snapshots of the jet penetration into gelatin. The laser is shot at $0 \mu \mathrm{s}$ and the subsequent images show the jet injection process at the designated times. The jet is created in a $500 \mu \mathrm{m}$ tube.

Figure 4 shows the penetration depth for the jets created in tubes with three different diameters. As discussed in [13, tubes with larger diameters result in jets with larger diameters. At the same time, the penetration depth increases with the diameter of the jet. For the $500 \mu \mathrm{m}$ tube case, the jet can penetrate up to $\sim 5 \mathrm{~mm}$ in a single shot, something that has never been achieved so far. For the $100 \mu \mathrm{m}$ tube case, the jet penetrates $0.5 \mathrm{~mm}$ at a velocity of $320 \mathrm{~m} / \mathrm{s}$, close to sonic speed. Thanks to the large velocity range of our jets, we can achieve the same penetration depth by using different tube diameters, which enables us to control the injection volume with nano-liter precision.

All data sets show a linear relation between the penetration depth and the jet velocity. Remarkably the viscous 


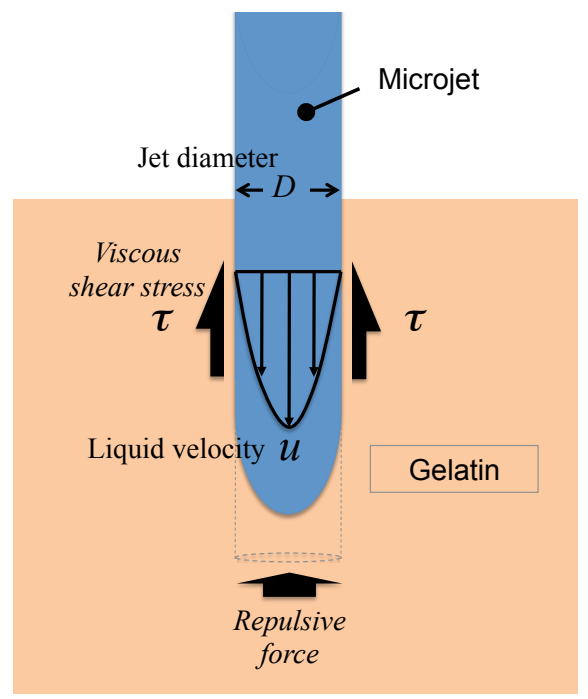

FIG. 2: The schematic sketch of the forces acting on the jet. The jet shown by the blue color region is penetrating into the gelatin. The viscous shear stress $\tau$ acts at the interface between liquid and gelatin due to the shear flow inside the jet. The repulsive force acts vertically on the projection area of the jet shown by the dashed closed line.

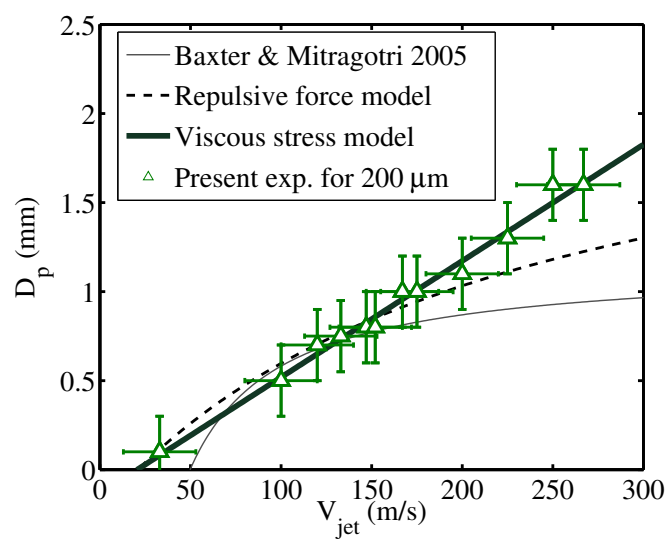

FIG. 3: Injection depth as a function of the jet velocity. Green triangles show the experimental results for the $200 \mu \mathrm{m}$ tube. The depth increases with the jet velocity and no saturation tendency is observed. For comparison, the gray thin line shows the Baxter model, the blue dashed line is the repulsive force model, and the black thick line is the viscous stress model with same offset. The experimental results agree well with this latter model.

stress model (equation 2) discussed above holds for all cases. We will now try to calculate the parameter $c_{v}$ in equation 22 from the jet geometry: We approximate the jet shape by a cylinder, whose mass $m_{j}=\pi \rho D^{2} l / 4$, where $l$ is the length of the jet cylinder. The total viscous stress on the jet is $F_{v}=\int_{d A} \tau d A$, where $A$ is the area on which the viscous shear stress acts. The elongated shape of the jet, i.e. the aspect ratio $D / l \ll 1$ allows us to approximate $A \approx \pi D l$, leading to $F_{v} \sim \mu v l$. Thus we obtain equation (1) $F_{D} \sim c_{v} \cdot v$ with $c_{v} \propto D^{-2}$, meaning that $c_{v}$ quadratically decreases with increasing $D$. The experimental values $c_{v}$ for each tube jet are shown in the inset in the figure 4 . Indeed, larger values for $c_{v}$ are found for smaller $D$. Assuming a power low $c_{v} \propto D^{\alpha}$ we obtain from the experimental results $\alpha=-1.35 \pm 0.48$, slightly larger than the model result $\alpha=-2$.

We also experimentally measure the time evolution of the penetration depth and compare it with the viscous stress model. The model leads to an exponential temporal evolution of the penetration depth $D_{p}(t)$,

$$
D_{p}(t)=\frac{v_{j e t}-v_{c}}{c_{v}}\left(1-e^{-c_{v} t}\right)
$$

The viscous stress model shows an agreement with the measurement within error bars as shown in figure 5 This result provides additional support in favor of the viscous stress model for gelatin. 


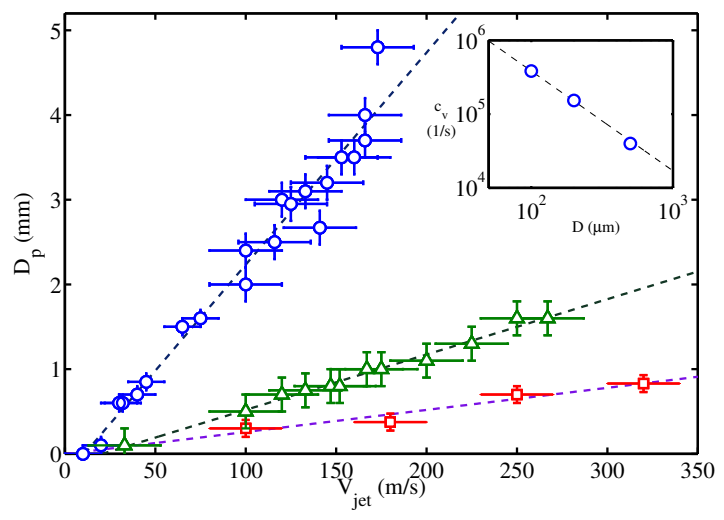

FIG. 4: Injection depth as a function of the jet velocity for different capillary tube diameters. The data for each capillary tube are fitted by the viscous stress model, shown by the dashed line. The fitted slope $c_{v}$ is plotted as a function of the capillary size in the inset. The data can be represented by $c_{v} \propto D^{-1.35 \pm 0.48}$, shown as the black dash-dotted line in the inset.

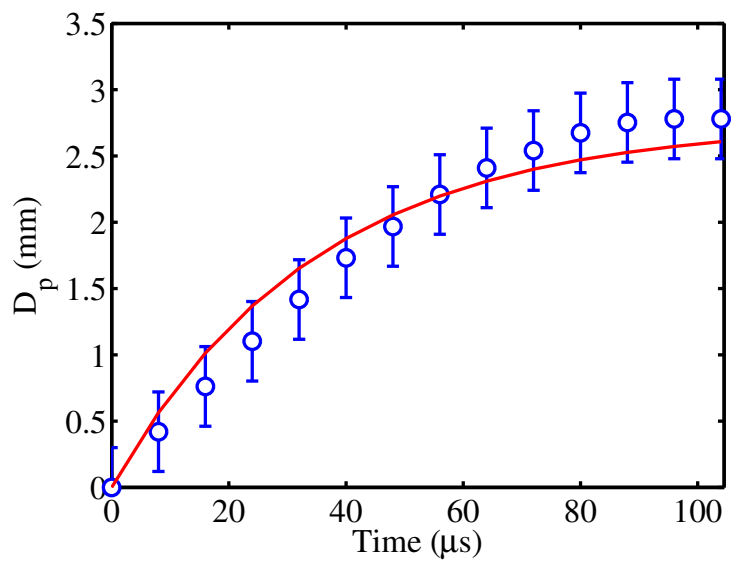

FIG. 5: The time evolution of the penetration depth $D_{p}$. The blue markers show the experimental results for the jet of $v_{j e t}$ $=120 \mathrm{~m} / \mathrm{s}$ for the $500 \mu \mathrm{m}$ tube. The red thick line shows the penetration depth from equation (5) with $c_{v}$ obtained by fitting the model by equation (2) to all data for the $500 \mu \mathrm{m}$ tube shown in figure 4

\section{B. Injection into artificially grown human skin}

In order to mimic the real human body, we have used artificially grown human skin placed on top of the gelatin 5 $\mathrm{wt} \%$ as a target material for our jets. Figure 6 shows snapshots of the jet penetrating into this material comprising of both the skin and the gelatin. The tip of the jet is observed for the first time in the gelatin at $46 \mu \mathrm{s}$. At this point, it is clear that the jet is able to penetrate human skin. After this stage, the penetration dynamics are similar to those in the gelatin case shown in figure 1. The jet is still focused even though the jet has to penetrate through the additional barrier of skin.

Figure $7(\mathrm{~b})$ shows the penetration depth $\left(D_{p}\right)$ into the gelatin through the skin as a function of the initial impact velocity $\left(\vec{\nabla}_{j e t}\right)$ of the jet. Note that the data only represent the penetration depth $\left(D_{p}\right)$ into the gelatin, excluding the skin thickness $\left(l_{s}\right)$ as indicated in figure 7 (a). The threshold velocity for penetrating through the skin is found to be $80 \mathrm{~m} / \mathrm{s}$. Even after the jet has penetrated an additional barrier in the form of human skin, the depth depends linearly on the initial velocity $\left(V_{j e t}\right)$. This suggests an excellent controllability of this system, which is crucial for medical applications. As seen in figure 7(b), the jet can penetrate more than a millimeter into the soft tissues (gelatin in the present case), after passing through the skin barrier. This depth is sufficient for most medical applications, e.g. 


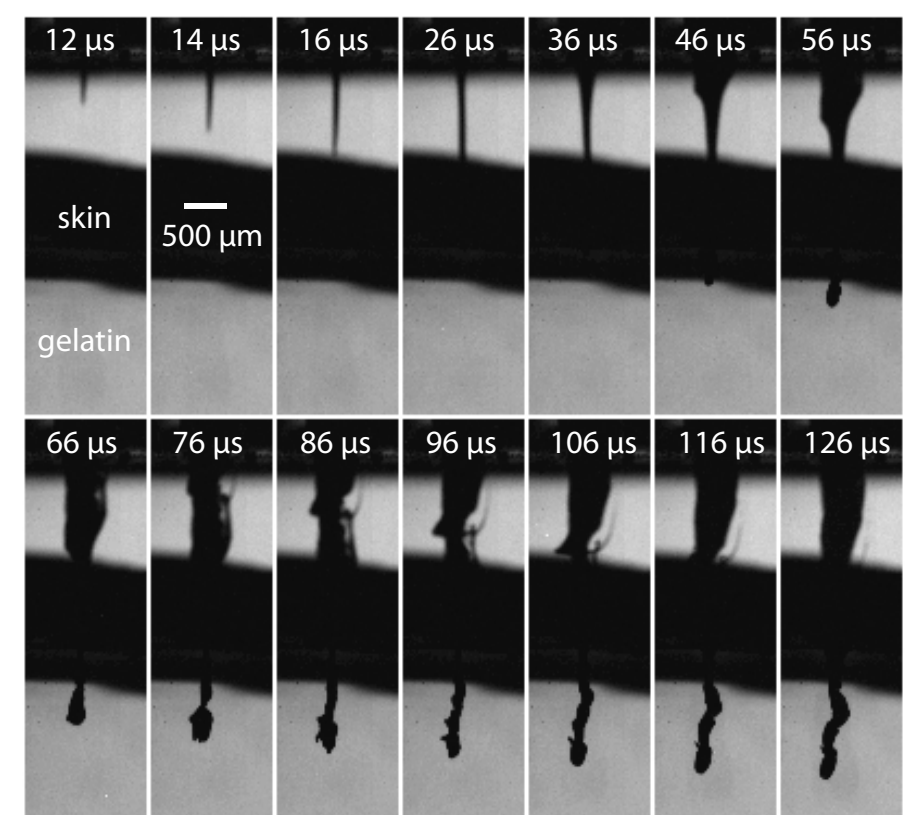

FIG. 6: Time evolution of the jet penetration into human skin placed on gelatin. After the second snapshot, the time interval for each image is $10 \mu \mathrm{s}$. The laser is shot at $0 \mu \mathrm{s}$. The jet impact velocity is $160 \mathrm{~m} / \mathrm{s}$. Note that the dark region of the skin in the images is thicker than the actual thickness of the skin, as the skin curls up on the sides of the cuvette.

(a)

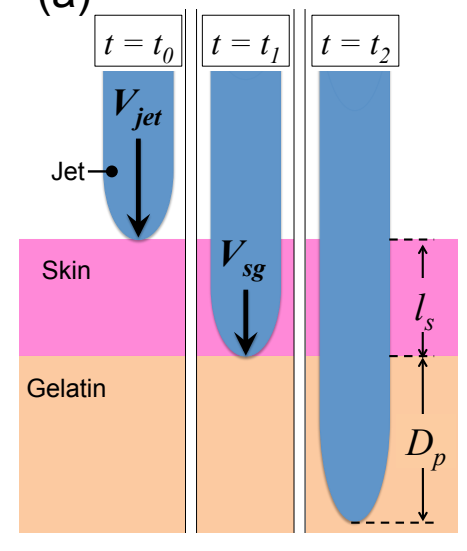

(b)

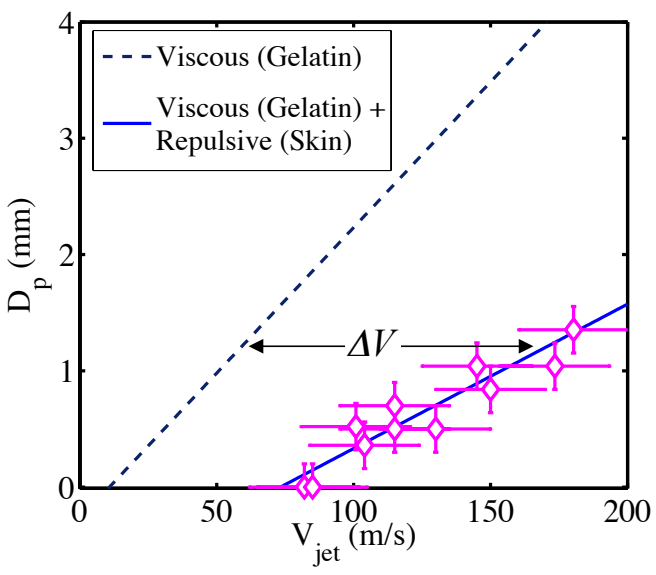

(c)

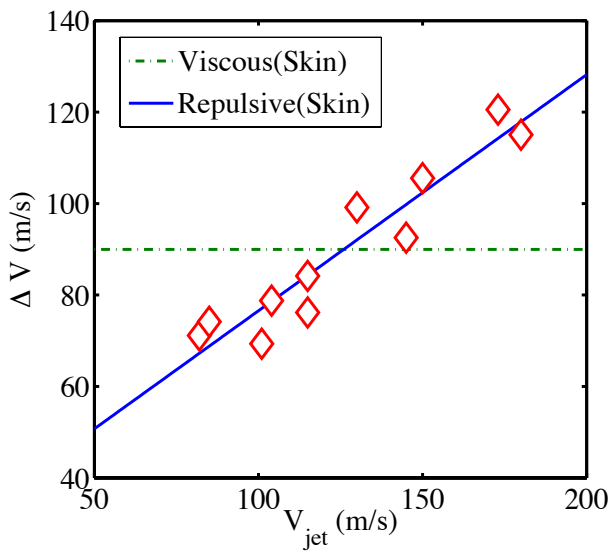

FIG. 7: (a) A sketch for the jet penetration into the gelatin covered with the artificially grown human skin layer (the thickness $\left.l_{s}=700 \mu \mathrm{m}\right)$. At $t=t_{0}$ the jet impacts the skin layer with the velocity $V_{j e t}$. At $t=t_{1}$ the jet goes through the skin layer and start penetrating the gelatin with the velocity $V_{s g}$. At $t=t_{2}$ the jet stops at the final depth $D_{p}$. (b) Diamonds: the final depth $D_{p}$ into gelatin with the skin layer as a function of the jet velocity. The dashed line: the case for gelatin without skin attached. The blue thick line: the results of the model represented by equation (8). (c) The velocity reduction $\Delta V$ due to the skin layer vs. the impact velocity. The dark green line: the viscous stress model for the skin, and the blue thick line: the repulsive force model (equation (7)).

insulin injection or vaccinations. When compared to the pure gelatin case (dashed line in figure 7(b)), the penetration depth is of course smaller with the skin layer being present. The skin decelerates the jet until complete penetration through itself (at $t=t_{1}$ as shown in figure 7(a)), after which the jet penetrates the gelatin until complete stoppage (at $t=t_{2}$ ). For the latter process we again adopt the viscous drag model, but with a reduced velocity $v_{s g}$ due to the additional barrier of the skin (as shown in figure 7(a)). The velocity reduction by the skin layer is

$$
\Delta V=V_{j e t}-V_{s g},
$$


which, as indicated in figure $7(\mathrm{~b})$, is equivalent to the horizontal offset between the line for the pure gelatin and the measured data with the skin layer being present. Figure 7 (c) shows this velocity reduction $(\Delta V)$ as a function of the impact velocity $\left(V_{\text {jet }}\right)$.

We evaluate the velocity reduction by considering the drag of the skin layer again with two different models: the viscous stress model and the repulsive force model. The viscous stress model for the skin layer leads to a constant velocity reduction for a given skin thickness (see equation 2). However, the experimental results show a different trend in figure 7 (c). Hence, we model the skin layer with a repulsive force (see equation 3), and the corresponding velocity reduction is

$$
\Delta V=v_{j e t}-\left(v_{j e t}-v_{s}\right) e^{-c_{i, s} l_{s}}
$$

with two fitting parameters: $c_{i, s}$ and $v_{s}$, and the skin thickness $l_{s}=700 \mu \mathrm{m}$. Figure 7 (c) reveals that the model described by equation (7) with $c_{i, s}=1.0 \cdot 10^{3} \mathrm{~m}^{-1}$ and $v_{s}=51.5 \mathrm{~m} / \mathrm{s}$ shows a good agreement with the experiments, suggesting that the jet experiences the repulsive force in the skin layer. This is probably due to the increased hardness of the skin compared to that of gelatin. The final penetration depth inside the gelatin can be therefore obtained as

$$
D_{p}=\frac{1}{c_{v}}\left(\left(v_{j e t}-v_{s}\right) e^{-c_{i, s} l_{s}}-v_{c}\right),
$$

which is plotted as the thick line in figure 7(b). The excellent agreement suggests that the model (equation (8)) combining the repulsive force for the skin layer and the viscous drag for the gelatin, nicely describes the depth of the jet penetration. This model is thus suited to quantitatively describe the penetration of high-speed jets into human skin enclosing soft tissue.

In this study we have shown that a novel method for needle-free injections can resolve many of the longstanding issues that have prevented largescale adaptation of needle-free injection systems. We show that a highly-focused geometry of the jets and a wide range of velocities is essential for good controllability, versatility, and effectiveness of needle-free injection systems. We also model the penetration of the jet into soft matter and human skin enclosing soft tissue. The results presented here take needle-free injections a step closer to widespread use.

\section{Materials}

\section{Microjet Generation}

The highly focused high-speed microjets are generated by focusing a laser pulse into a small capillary tube filled with water-based red dye. This leads to the abrupt vaporization of a small mass of liquid [20]. The vaporization causes a shock wave to travel through the liquid and impulsively accelerate the curved liquid interface due to kinematic focusing. The capillary tube is connected to a syringe through micro tubing and the dye is pumped into the capillary tube using a syringe pump. The characteristics of this jet, such as velocity and width, can be controlled by varying the laser power, the distance between the laser focus and the free surface, the liquid-glass contact angle, and the diameter of the tube $[13]$.

\section{Injection Into Gelatin}

Gelatin mixtures were used to study the injection into solid substrates. The gelatin was prepared a few hours before the experiments by dissolving 5 weight $\%$ of gelatin in MilliQ water. After dissolving the gelatin, the mixture was poured into small $1 \mathrm{~cm} \times 1 \mathrm{~cm}$ cuvettes and put in the fridge $\left(4^{\circ} \mathrm{C}\right)$ for an hour. Penetration dynamics were filmed using high-speed cameras with frame rates up to $10^{6}$ fps (HPV-1, Shimadzu Corporation, Japan, and FASTCAM SAX, Photron, USA).

\section{Penetration Across Human Skin In Vitro}

The artificial skin was cultured by the Department of Dermatology of the Leiden University Medical Center. The Leiden Human Epidermal (LHE) skin model used in this study has been fully characterized and shows very high similarities with native skin [21]. The LHE represents a full-thickness model (epidermis generated onto a dermal 
matrix). The skin was supplied in patches of $2.4 \mathrm{~cm}$ in diameter and was kept in an incubator prior to experiments. For the penetration experiments, the skin layers were placed on top of the gelatin mixtures in the small cuvettes. Penetration dynamics were filmed using high-speed cameras (HPV-1, Shimadzu Corporation, Japan, and FASTCAM SAX, Photron, USA).

\section{Velocity and Depth Measurement}

High-speed cameras (HPV-1, Shimadzu Corporation, Japan, and FASTCAM SAX, Photron, USA) were used to record the injection process. The velocity and depth were determined from these high-speed recordings.

\section{Acknowledgments}

We thank C. Clanet, F. Dijksman, B. Hoeksma, L. Homan, Devaraj van der Meer, Vivek N. Prakash, and C.W. Visser for fruitful discussions. We appreciate the financial support given by Fundamenteel Onderzoek der Materie (FOM), which is part of Nederlandse Organisatie voor Wetenschappelijk Onderzoek (NWO) and the European Research Council (ERC) through a Proof of Concept Grant.

[1] A. Kane, J. Lloyd, M. Zaffran, L. Simonsen, and M. Kane, Transmission of hepatitis B, hepatitis C and human immunodeficiency viruses through unsafe injections in the developing world: model-based regional estimates, Bull. World Health Organ., 77 (1999), pp. 801-807.

[2] H. Varmus, R. Klausner, E. Zerhouni, T. Acharya, A.S. Daar, and P.A. Singer, Grand Challenges in Global Health, Science, 17 (2003), 398-399.

[3] A.M. Hauri, G.L. Armstrong, and Y.J.F. Hutin, The global burden of disease attributable to contaminated injections given in health care settings, Int. J. STD AIDS, 15 (2004), pp. 7-16;

[4] M. Kermode, Unsafe injections in low-income country health settings: need for injection safety promotion to prevent the spread of blood-borne viruses, Health Promot. Int., 19 (2004), pp. 95-103.

[5] Y. Nir, A. Paz, E. Sabo, and I. Potsman, Fear of Injection in Young Aduls: Prevalence and Associations, Am. J. Trop. Med. Hyg., 68 (2003), pp. 341-344

[6] S. Mitragotri, Immunization without needles, Nature Rev. Immunol., 5 (2005), pp. 905-916.

[7] A. Arora, I. Hakim, J. Baxter, R. Rathnasingham, R. Srinivasan, D.A. Fletcher, and S. Mitragotri, Needle-free delivery of macromolecules across the skin by nanoliter-volume pulsed microjets, Proc. Nat. Acad. Sci., 104 (2007), pp. 4255-4260.

[8] V. Menezes, S. Kumar, and K. Takayama, Shock wave driven liquid microjets for drug delivery, J. Appl. Phys., 106 (2009), 086102.

[9] J.C. Stachowiak, T.H. Li, A. Arora, S. Mitragotri, D.A. Fletcher, Dynamic control of needle-free jet injection, J. Controlled Release, 135 (2009), pp. 104-112.

[10] S. Mitragotri, Current status and future prospects of needle-free liquid jet injectors, Nature Rev. Drug Discovery, 5 (2006), pp. 543-548.

[11] J. Baxter and S. Mitragotri, Jet-induced skin puncture and its impact on needle-free jet injections: experimental studies and a predictive model, J. Controlled Release, 106 (2005), pp. 361-373.

[12] J. Schramm-Baxter and S. Mitragotri, Needle-free jet injections: dependence of jet penetration and dispersion in the skin on jet power, J. Controlled Release, 97 (2004), pp. 527-535.

[13] Y. Tagawa, N. Oudalov, C.W. Visser, I.R. Peters, D. van der Meer, C. Sun, A. Prosperetti, and D. Lohse, Highly Focused Supersonic Microjets, Phys. Rev. X, 2 (2012), 031002.

[14] N.C. Hogan, B.D. Hemond, D.M. Wendell, A.J. Taberner, and I.W. Hunter, Delivery of Active Collagenase to Skin Using a Lorentz-Force Actuated Needle-Free Injector, Proceedings of the 28th IEEE EMBS Annual International Conference, New York City, USA, (2006).

[15] C. Weller, and M. Linder, Jet injection of insulin vs the syringe-and-needle method, JAMA, 195 (1966), pp. 844-847.

[16] D.L. Bremseth, and Franklin Pass, Delivery of Insulin by Jet Injection: Recent Observations, Diabetes Technology \& Therapeutics, 3 (2001), pp. 225-232.

[17] B.G. Weniger, Jet Injection of Vaccines: Overview and Challenges for Mass Vaccination with Jet Injections, in Innovative Administration Systems for Vaccines, (2003), Rockville, Maryland.

[18] E.L. Giudice, and J.D. Campbell, Needle-free vaccine delivery, Advanced Drug Delivery Reviews, 58 (2006), pp. 68-89.

[19] M.A.F. Kendall, Needle-Free Vaccine Injection, Handbook of Experimental Pharmacology: Drug Delivery, 197 (2010), pp. 193-219. 
[20] C. Sun, E. Can, R. Dijkink, D. Lohse, and A. Prosperetti, Growth and Collapse of a Vapour Bubble in a Microtube: The Role of Thermal Effects, J. Fluid Mech., 632 (2009), pp. 5-16.

[21] A. El Ghalbzouri, R. Siamari, R. Willemze, and M. Ponec, Leiden reconstructed human epidermal model as a tool for the evaluation of the skin corrosion and irritation potential according to the ECVAM guidelines, Toxicology in Vitro, 97 (2008), pp. 1311-1320. 